\title{
Analysis of the role of the Cronobacter sakazakii ProP homologues in osmotolerance
}

\author{
Audrey Feeney, Christopher D Johnston, Rodney Govender, Jim O'Mahony, Aidan Coffey and Roy D Sleator
}

\begin{abstract}
Bacteria respond to elevated osmolality by the accumulation of a range of low molecular weight molecules, known as compatible solutes (owing to their compatibility with the cells' normal physiology at high internal concentrations). The neonatal pathogen Cronobacter sakazakii is uniquely osmotolerant, surviving in powdered infant formula (PIF) which typically has a water activity $\left(a_{w}\right)$ of 0.2 - inhospitable to most micro-organisms. Mortality rates of up to $80 \%$ in infected infants have been recorded making C. sakazakii a serious cause for concern. In silico analysis of the C. sakazakii BAA-894 genome revealed seven copies of the osmolyte uptake system ProP. Herein, we test the physiological role of each of these homologues following heterologous expression against an osmosensitive Escherichia coli host.
\end{abstract}

Keywords: Osmolytes, Proline, Osmotolerance, Stress, Cronobacter

\section{Introduction}

Cronobacter spp. (previously Enterobacter sakazakii) are Gram-negative, motile, facultatively anaerobic foodbourne pathogens belonging to the Enterobacteriacea family [1]. The Cronobacter genus, which has become increasingly diverse in recent years, comprises ten species: Cronobacter sakazakii, Cronobacter malonaticus, Cronobacter turicensis, Cronobacter universalis, Cronobacter muytjensii, Cronobacter dublinensis, Cronobacter condiment, Cronobacter pulveris, Cronobacter helveticus and Cronobacter zurichensis [2,3]. Cronobacter sakazakii is an opportunistic pathogen capable of causing severe clinical symptoms in adults, infants and elderly patients (FAO/WHO 2008). The most at risk group are pre-term, low birth weight neonates who can suffer life-threatening necrotizing enterocolitis, meningitis and septeceamia, with resulting mortality rates of $40-80 \%$ [4]. In a recent study multilocus sequence typing revealed that the majority of meningitis cases in neonates are caused as a result of infection with one clonal lineage, C. sakazakii ST4 clonal complex [2]. While a significant proportion of infants succumb to the infection within days of birth [5], survivors often suffer severe neurological damage [6].

\footnotetext{
* Correspondence: roy.sleator@cit.ie
Department of Biological Sciences, Cork Institute of Technology, Rossa

* Correspondence: roy.sleator@cit.ie
Department of Biological Sciences, Cork Institute of Technology, Rossa Avenue, Bishopstown, Cork, Ireland
}

(C) 2014 Feeney et al.; licensee BioMed Central Ltd. This is an Open Access article distributed under the terms of the Creative Commons Attribution License (http://creativecommons.org/licenses/by/4.0), which permits unrestricted use, distribution, and reproduction in any medium, provided the original work is properly credited. The Creative Commons Public Domain Dedication waiver (http://creativecommons.org/publicdomain/zero/1.0/) applies to the data made available in this article, unless otherwise stated.
While studies have identified plant material (in particular vegetables, fruits, cereals and rice) as the most likely source, foods such as wheat, cheese, meat, herbs and spices have also been found to harbour the pathogen [7]. However, it is the presence of $C$. sakazakii in powdered infant formula (PIF) which is cause for most concern (FAO/WHO 2008). Indeed, PIF has been epidemiologically linked with several major outbreaks of C. sakazakii infection [8]. Although isolated at low levels, persistence of the pathogen for extended periods, in some cases for up to 2.5 years [9] makes $C$. sakazakii a particularly formidable pathogen. Given that pasteurisation has been shown to effectively inactivate $C$. sakazakii, the presence of the organism in PIF can be attributed to contamination resulting from the addition of contaminated ingredients and/or the use of non-sterile equipment either during processing or reconstitution [10].

Survival of C. sakazakii in PIF, which has an $\mathrm{a}_{\mathrm{w}}$ of $\sim 0.2$ (significantly below the normal cut off of 0.4 for canonical microbial growth), suggests that the pathogen is uniquely armed with an extensive array of stress survival mechanisms necessary to overcome this otherwise inhospitable environment [11]. Indeed, a previous in silico analysis of C. sakazakii BAA-894 (a strain originally isolated from powdered infant formula [12]) revealed 53 putative osmotolerance loci, including both hyper- and hypo-osmotic stress response systems. Interestingly, while C. sakazakii harbours homologues of all the principal osmotolerance 
loci of Escherichia coli; a key distinguishing feature is that C. sakazakii possesses multiple copies of certain osmotolerance mechanisms; including seven copies of the $E$. coli proP homologue, each of which potentially encodes a separate osmolyte uptake system.

Herein, we describe the functional analysis of the putative osmotolerance loci ESA_02131, ESA_01706, ESA_04214, ESA_pESA3p05450, ESA_00673 and ESA_03328, when heterologously expressed against E. coli MKH13 (an osmotically sensitive mutant). Furthermore, given that the $C$. sakazakii osmotic stress response is likely regulated at the level of DNA expression [11], we analysed the transcriptional response of each of the proP homologues following osmotic up-shock.

\section{Materials and methods}

\section{Bacterial strains, plasmids and growth conditions}

Bacterial strains and plasmids used in this study are listed in Table 1. C. sakazakii BAA-894 and E. coli DH5 $\alpha$ clones were grown at $37^{\circ} \mathrm{C}$ in LB broth (Sigma-Aldrich Co.). E. coli MKH13 strains were grown in LB or M9 minimal medium containing $0.5 \%$ glucose, $0.04 \%$ arginine, $0.04 \%$ isoleucine, $0.04 \%$ valine (Sigma-Aldrich Co.). After filter sterilization, proline or glycine betaine (Sigma-Aldrich Co.) was added to M9 to a final concentration of $1 \mathrm{mM}$. Ampicillin was made up as a concentrated stock solution and added to media at the required levels. Medium osmolarity was adjusted using $\mathrm{NaCl}$.

\section{In silico analysis of $C$. sakzakii BAA-894}

Putative proP loci were identified using a homology based transfer approach, as described previously [11]. Further in silico analysis was carried out with the aid of the Lasergene suite of applications (DNASTAR, Madison, USA) and Simplicity [16] (nSilico, Cork, Ireland).

\section{DNA manipulations and sequence analysis}

Genomic DNA was extracted from C. sakazakii as described by Sambrook et al. [17]. Plasmid DNA was isolated with the High Pure Plasmid Isolation Kit (Roche Diagnostics). PCR primers (Table 2) were designed for each proP homologue based on C. sakazakii strain BAA-894 sequence data available from the NCBI database (NC_009778.1). All PCR reactions were carried out using the high fidelity Velocity DNA polymerase Kit (Bioline) in accordance with manufacturer's instructions. The $\mathrm{T} a$ of each reaction was dependent on the Tm of individual primers. Negative control reaction mixtures were included with sterile nuclease free water in place of template DNA. Restriction enzymes and T4 DNA ligase were purchased from Roche Diagnostics (Mannheim, Germany). Each restriction digest and T4 ligation reaction was carried out as per the manufacturer's

Table 1 Bacterial strains and plasmids

\begin{tabular}{|c|c|c|}
\hline Strain or plasmid & Relevant genotype or characteristics & Source or reference \\
\hline \multicolumn{3}{|l|}{ Plasmids } \\
\hline pUC18 & Amp', lacZ', pMB9 replicon & {$[13]$} \\
\hline pUC18::ESA_02131 & pUC18 with ESA_02131 gene under control of native promoter & This work \\
\hline pUC18::ESA_01706 & pUC18 with ESA_01706 gene under control of native promoter & This work \\
\hline pUC18::ESA_04214 & pUC18 with ESA_04214 gene under control of native promoter & This work \\
\hline pUC18::ESA_pESA3p05450 & pUC18 with ESA_pESA3p05450 gene under control of native promoter & This work \\
\hline pUC18::ESA_01226 & pUC18 with ESA_01226 gene under control of native promoter & This work \\
\hline pUC18::ESA_00673 & pUC18 with ESA_00673 gene under control of native promoter & This work \\
\hline pUC18::ESA_03328 & pUC18 with ESA_03328 gene under control of native promoter & This work \\
\hline \multicolumn{3}{|l|}{ Strains } \\
\hline Cronobacter sakazakii BAA-894 & C.sakazakii strain isolated from powdered formula associated with neonatal intensive care unit & {$[12]$} \\
\hline Escherichia coli DH5a & Intermediate cloning host.supE44 $\Delta$ lacU169(80lacZDM15)R17 recA1 endA1 gyrA96 thi-1 relA1. & Invitrogen. \\
\hline MKH13 & MC4100 $\Delta($ putPA) $101 \Delta($ proP) $2 \Delta($ proU $)$ & {$[15]$} \\
\hline MKH13 pUC18::ESA_02131+ & Host strain harbouring pUC18: ESA_02131 plasmid. Ampr. & This work \\
\hline MKH13 pUC18::ESA_01706+ & Host strain harbouring pUC18: ESA_01706 plasmid. Ampr. & This work \\
\hline MKH13 pUC18::ESA_04214+ & Host strain harbouring pUC18: ESA_04214 plasmid. Ampr. & This work \\
\hline MKH13 pUC18::ESA_pESA3p05450+ & Host strain harbouring pUC18: ESA_pESA3p05450 plasmid. Ampr. & This work \\
\hline MKH13 pUC18::ESA_01226+ & Host strain harbouring pUC18: ESA_01226 plasmid. Ampr. & This work \\
\hline MKH13 pUC18::ESA_00673+ & Host strain harbouring pUC18: ESA_00673 plasmid. Ampr. & This work \\
\hline MKH13 pUC18::ESA_03328+ & Host strain harbouring pUC18: ESA_03328 plasmid. Ampr. & This work \\
\hline
\end{tabular}

$A m p^{r}$ - This strain is resistant to ampicillin. 
Table 2 Primers

\begin{tabular}{|c|c|c|c|c|}
\hline Primer name & & Primer sequence (5' to $\left.3^{\prime}\right)$ & Length & Characteristics \\
\hline \multirow[t]{2}{*}{ ESA_02131 } & $\mathrm{F}$ & CATCGGCCGACAGGCCAGTCAATGAATGATGC & 32 & Eag1cut site \\
\hline & $\mathrm{R}$ & CATTCTAGAGAGTACAACGGAATGCGGGG & 29 & Xba1 cut site \\
\hline \multirow[t]{2}{*}{ ESA_01706 } & $\mathrm{F}$ & CATTCTAGAGTCGGGCGGCTCTTTATCTGG & 30 & Xba1 cut site \\
\hline & $\mathrm{R}$ & CATGGATCCTTGACCAGATGACGCAGTCTTTC & 32 & BamH1 cut site \\
\hline \multirow[t]{2}{*}{ ESA_04214 } & $\mathrm{F}$ & CATGAATTCGTCTCTITCTGTGCCAACTATCTGC & 34 & EcoR1 cut site \\
\hline & $\mathrm{R}$ & CATTCTAGACTACCTGACGCGTACCCTGTATATC & 34 & Xba1 cut site \\
\hline \multirow[t]{2}{*}{ ESA_pESA3p05450 } & $\mathrm{F}$ & CATGAATTCATCATCTCTACACGCTGCCTTCTG & 33 & EcoR1 cut site \\
\hline & $\mathrm{R}$ & CATTCTAGATCTCCACCTGCGCCTCTATC & 29 & Xba1 cut site \\
\hline \multirow[t]{2}{*}{ ESA_01226 } & $\mathrm{F}$ & CATGAATTCCAGTGCGCCGGAGCTTTTCG & 29 & EcoR1 cut site \\
\hline & $\mathrm{R}$ & CATTCTAGAGGGCTGTCGGTTGACGAAATTAAAC & 34 & Xba1 cut site \\
\hline \multirow[t]{2}{*}{ ESA_00673 } & $\mathrm{F}$ & CATGAATTCTAAAAGCGAAATCCTCCCGTACTGGC & 35 & EcoR1 cut site \\
\hline & $\mathrm{R}$ & CATGGATCCCCTGCAAAGCATCGCCGATTACC & 32 & BamH1 cut stie \\
\hline \multirow[t]{2}{*}{ ESA_03328 } & $\mathrm{F}$ & CATTCTAGATCGCTATCGCTGACCGTGAAATG & 32 & Xba1 cut site \\
\hline & $\mathrm{R}$ & CATGGATCCTGCTGAACGAACAGTATGGCCG & 31 & BamH1 cut site \\
\hline \multirow[t]{2}{*}{ pUC18 MCS Check } & $\mathrm{F}$ & CATTAG CTC ACT CAT TAG GCA CC & 20 & pUC18 insert check \\
\hline & $\mathrm{R}$ & CATTGT AAA ACG ACG GCC AGT G & 19 & pUC18 insert check \\
\hline \multirow[t]{2}{*}{ RTESA_02131 } & $\mathrm{F}$ & GCTGGCGTGTATCGGTCT & 18 & Probe 31 \\
\hline & $\mathrm{R}$ & CTC GGC ATA TAA GTA AGC AGC AT & 23 & Probe 31 \\
\hline \multirow[t]{2}{*}{ RTESA_01706 } & $\mathrm{F}$ & CGA CGG TCA TTC TGC TCA C & 19 & Probe 32 \\
\hline & $\mathrm{R}$ & AGC AGG CCA ATC TGA TGG TA & 20 & Probe 32 \\
\hline \multirow[t]{2}{*}{ RTESA_04214 } & $\mathrm{F}$ & GCA TAA ACG CGC CCT GTA & 18 & Probe 18 \\
\hline & $\mathrm{R}$ & TTA GCG AAG AAG AAG CCG ATA & 21 & Probe 18 \\
\hline \multirow[t]{2}{*}{ RTESA_01226 } & $\mathrm{F}$ & GGG TAT CAG GTG GCA AGC & 18 & Probe 70 \\
\hline & $\mathrm{R}$ & GTA AAT CGC GAC CGT ATG C & 19 & Probe 70 \\
\hline \multirow[t]{2}{*}{ RTESA_00673 } & $\mathrm{F}$ & CCG GAC AGA TAA ACC GTC AC & 20 & Probe 11 \\
\hline & $\mathrm{R}$ & GAC GAG GCA CCG ACA ATC & 18 & Probe 11 \\
\hline \multirow[t]{2}{*}{ RTESA_03328 } & $\mathrm{F}$ & CGT GCC GTT CGT AAT GGT & 18 & Probe 29 \\
\hline & $\mathrm{R}$ & AAC GAA ATG CCG GTA AAG C & 19 & Probe 29 \\
\hline \multirow[t]{2}{*}{ RTESA_pESA3p05450 } & $\mathrm{F}$ & TGG TGG CGA TIT CCA ACT & 18 & Probe 71 \\
\hline & $\mathrm{R}$ & CCC GAA ACG GTC AGA AAG & 18 & Probe 71 \\
\hline \multirow[t]{2}{*}{ RT16S } & $\mathrm{F}$ & TGT AGC GGT GAA ATG CGT AG & 20 & Probe 65 \\
\hline & $\mathrm{R}$ & AGC GTC AGT CTT CGT CCA G & 19 & Probe 65 \\
\hline
\end{tabular}

instructions within $0.2 \mathrm{ml}$ PCR tubes (Sarstedt) in a thermocycler block to ensure optimal temperature conditions. Plasmid pUC18 was used as the cloning platform; with individual vectors being constructed for the expression of each gene under the control of its own native promoter. The integrity of each C. sakazakii gene was confirmed by sequencing (MWG Operon, Germany and GATC, Germany). Electrocompetent E. coli DH5 $\alpha$ were obtained from New England Biolabs and E. coli MKH13 was made electrocompetent using the method outlined by Sambrook and Russell [17]. Electrotransformation was carried out using standard methods.

\section{Gene expression analysis (qRT-PCR)}

Total RNA was isolated (Roche High Pure RNA Isolation Kit) from C. sakazakii grown in a shaking incubator at $37^{\circ} \mathrm{C}$ prior to osmotic shock and up to 2 hours post shock with $6 \% \mathrm{NaCl}$. RNA quantity and quality was confirmed by microphotometry (Nanodrop, De, USA). Complementary DNA (cDNA) was synthesised using $1 \mu \mathrm{g}$ total RNA incubated with $50 \mathrm{pM}$ of random hexamer primers followed by a $20 \mu \mathrm{l}$ reverse transcription reaction using the Bioloine cDNA synthesis kit, as per manufacturer's instructions (Bioline). The amplification conditions used were $42^{\circ} \mathrm{C}$ for 1 hour, $70^{\circ} \mathrm{C}$ for $15 \mathrm{~min}$ and $4^{\circ} \mathrm{C}$ for $5 \mathrm{~min}$. PCR primers 
and probes were designed using the Universal Probe Library Assay Design Centre (http://lifescience.roche.com/ shop/home) and are summarised in Table 2. Optimisation of the gene expression assay was carried out on each primer/probe set by varying the concentration of either primer or probe to achieve the best signal. Based on these results, individual primer/probe mixes were made for each gene of interest. Amplification reactions contained $5 \mu \mathrm{l}$ cDNA, $10 \mu \mathrm{l}$ of the Light Cycler 480 probes Master 2x conc (Roche) and $2 \mu \mathrm{l}$ primer/probe mix in a total reaction of $20 \mu \mathrm{l}$. All reactions were performed in triplicate using 96 well plates on the Roche LightCycler 480 Real-Time PCR system. Thermal cycling conditions were as recommended by the manufacturer. The $2^{\Delta}{ }^{\Delta} \mathrm{CT}$ method was used to calculate relative changes in gene expression determined from these real time quantitative PCR experiments [18].

\section{Physiological analysis of $E$. coli MKH13 clones in media supplemented with $\mathrm{NaCl}$}

Each E. coli MKH13 clone expressing a different proP homologue (ESA_02131, ESA_01706, ESA_04214, ESA_pESA3p05450, ESA_00673 or ESA_03328) was grown overnight at $37^{\circ} \mathrm{C}$ in $10 \mathrm{mls}$ of $\mathrm{LB}$, or minimal media supplemented with the required components. The cells were pelleted by centrifugation at 5,000 g, washed and resuspended in $200 \mu \mathrm{l}$ ringers. The cell suspension was added to the appropriate filter sterilized media with varying concentrations $(0-10 \%)$ of added $\mathrm{NaCl}$. Growth was monitored in the relevant media over a 48 hour period and the optical density (OD) was measured at $600 \mathrm{~nm}$. Triplicate readings were taken and graphs were constructed using SigmaPlot 11.0.

\section{Results}

\section{Functional complementation of E. coli MKH13 with} proP homologues

Each of the proP homologues were amplified using primers with engineered cut sites flanking the gene of interest. PCR products were digested and ligated to similarly digested pUC18 plasmid. Each pUC18::proP plasmid was transformed to MKH13 following passage through an intermediate cloning host (DH5 $\alpha)$. The osmosensitive $E$. coli strain MKH13 was used to functionally screen each of the proP homologues for their osmotolerance potential. Unlike the parent strain, MC 4100, E. coli MKH13 is deficient in the transport systems PutP, ProP and ProU; rendering it incapable of growth in high osmolarity media $(\geq 4 \%$ added $\mathrm{NaCl}$ ). Six of the seven proP homologues were successfully transformed into E.coli MKH13 (ESA_01226 alone failed to be transformed and expressed in E.coli MKH13, despite repeated transformation attempts). Successful transformants were screened for osmotolerance by plating on LB agar and on defined media agar plates (supplemented with proline or glycine betaine to a final concentration of $1 \mathrm{mM}$ ) containing 4-10\% added $\mathrm{NaCl}$. No colonies appeared following a control transformation with pUC18 alone, while transformation efficiencies of $\sim 60 \mathrm{CFU} / \mu \mathrm{g}$ DNA were achieved for each of proP homologue constructs, with colonies appearing within 72 hours at $37^{\circ} \mathrm{C}$. Plasmids were extracted and retransformed into $E$. coli $\mathrm{MKH} 13$ to confirm complementation and inserts were confirmed by PCR and sequence analysis.

\section{proP expression analysis in C. sakazakii BAA-894}

The level of gene expression was measured relative to the constitutively expressed 16 s gene (ESA_03798). E. coli MKH13 expressing proP homologues ESA_02131, ESA_01706, ESA_04214, ESA_00673, ESA_03328 and ESAp_3p05450 exhibited an increase in expression levels in complex media (LB) following osmotic upshock with $6 \% \mathrm{NaCl}$ (Figure 1). Strains expressing ESA_02131 and ESA_04214 exhibited the highest increase in expression 1 hour post initial osmotic shock (19-fold and 47-fold increase respectively). However, maximum levels of expression were reached 30 minutes after initial osmotic shock in E. coli MKH13::01706 (3 fold increase), E. coli MKH13::00673 (5 fold increase), E.coli MKH13::03328 (2 fold increase) and E. coli MKH13:: pESA3p05450 (4 fold increase) (Table 3). This suggests a biphasic response at the transcriptional level; a rapid low level up regulation followed by a slower high level response.

\section{Physiological analysis of proP homologues expressed in E. coli MKH13}

Physiological analysis of each E. coli MKH13 strain transformed with a proP homologue was performed to

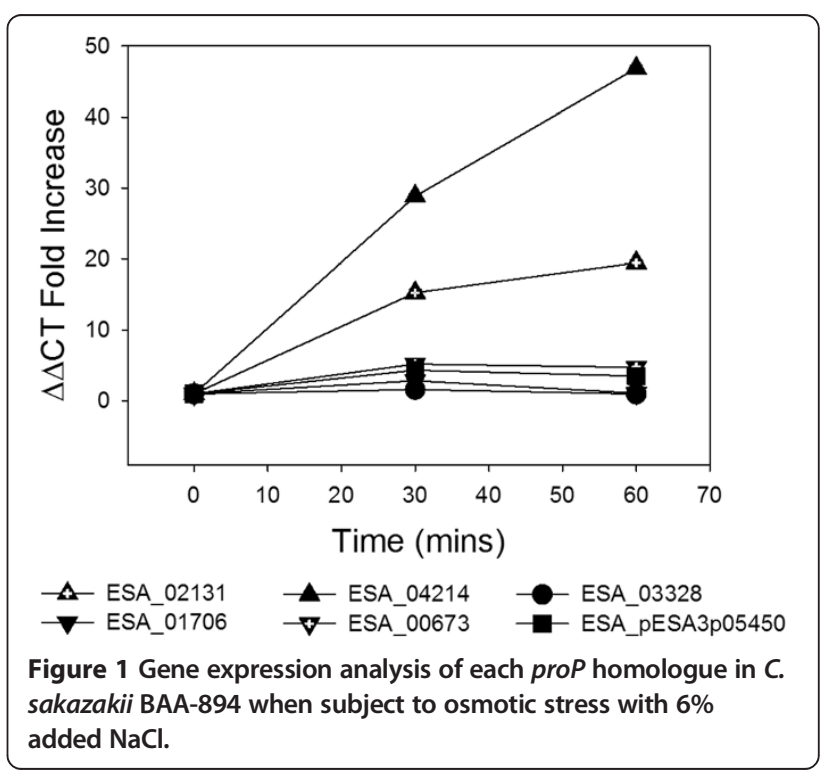


Table 3 Molecular and physiological comparison of each ProP homologue

\begin{tabular}{|c|c|c|c|c|c|c|c|c|}
\hline \multirow[t]{2}{*}{ Gene locus tag } & \multirow[t]{2}{*}{ Identity (\%) } & \multirow[t]{2}{*}{$\begin{array}{c}\text { Fold increase } \\
\text { in mRNA transcript }\end{array}$} & \multicolumn{2}{|c|}{ Complex media } & \multicolumn{2}{|c|}{$\begin{array}{l}\text { Defined media } \\
\text { supplemented } \\
\text { with proline }\end{array}$} & \multicolumn{2}{|c|}{$\begin{array}{l}\text { Defined media } \\
\text { supplemented with } \\
\text { glycine betaine }\end{array}$} \\
\hline & & & $\begin{array}{c}\text { Maximum } \\
\text { OD @ } 600 \mathrm{~nm}\end{array}$ & $\begin{array}{l}\text { Growth } \\
\text { rate }\left(\mathrm{hr}^{-1}\right)\end{array}$ & $\begin{array}{c}\text { Maximum } \\
\text { OD @ } 600 \mathrm{~nm}\end{array}$ & $\begin{array}{l}\text { Growth } \\
\text { rate }\left(\mathrm{hr}^{-1}\right)\end{array}$ & $\begin{array}{c}\text { Maximum } \\
\text { OD @ } 600 \mathrm{~nm}\end{array}$ & $\begin{array}{l}\text { Growth } \\
\text { rate }\left(\mathrm{hr}^{-1}\right)\end{array}$ \\
\hline ESA_02131 & 90 & 19.472 & 0.668 & 0.013 & 0.219 & 0.007 & 0.585 & 0.017 \\
\hline ESA_01706 & 40 & 2.854 & 0.259 & 0.000 & 0.205 & 0.008 & 0.358 & 0.011 \\
\hline ESA_04214 & 33 & 46.851 & 0.240 & 0.001 & 0.178 & 0.000 & 0.240 & 0.000 \\
\hline ESA_03328 & 30 & 1.598 & 0.255 & 0.003 & 0.261 & 0.021 & 0.397 & 0.021 \\
\hline ESA_00673 & 29 & 5.193 & 0.505 & 0.013 & 0.125 & 0.000 & 0.273 & 0.000 \\
\hline ESA_pESA3p05450 & 29 & 4.327 & 0.291 & 0.003 & 0.170 & 0.003 & 0.235 & 0.002 \\
\hline
\end{tabular}

The $\%$ identity values were obtained in a previous study [11] and determined the homology of the above $C$. sakazakii putative proP genes relative to the well characterised E. coli proP. Growth rate, OD and transcription values shown here were obtained when growth was monitored in $6 \%$ added $\mathrm{NaCl}$.

determine if each homologue conferred osmotolerance on the heterologous host. Growth of E. coli MKH13 was monitored over a 48 hour period in complex (LB) and defined media with varying concentrations of $\mathrm{NaCl}(0-10 \%$ added $\mathrm{NaCl}$ ). E. coli MKH13 expressing the empty pUC18 vector was used as a negative control; failing to grow at $\mathrm{NaCl}$ concentrations $\geq 4 \%$ added $\mathrm{NaCl}$.

\section{a) Growth in complex media at elevated osmolarity}

Each of the six strains expressing a proP homologue grew in $\mathrm{LB}$ broth supplemented with $\geq 4 \%$ added $\mathrm{NaCl}$, with the control strain (E. coli MKH13::pUC18) exhibiting no growth. At the most discriminatory salt concentration $(6 \%$ added $\mathrm{NaCl})$ growth was observed in five of the strains tested but to varying degrees (Table 3). However, above 6\% $\mathrm{NaCl}$ only strains expressing ESA_00673, ESA_02131 and ESA_pESA3p05450 demonstrated an ability to grow. ESA_00673 conferred the highest osmotolerance with a growth rate of $0.007 \mathrm{~h}^{-1}$ at $8 \% \mathrm{NaCl}$ and a maximum OD of $0.57 @ 600$ nm was recorded after 12 hours incubation at $37^{\circ} \mathrm{C}$. The next most osmotolerant strains in complex media at elevated osmolarity were those expressing ESA_02131 and the plasmid encoded ESA_pESA3p05450. Both of these strains grew in $\mathrm{LB}$ containing up to $7 \%$ added $\mathrm{NaCl}$. At this concentration E. coli MKH13 expressing ESA_02131 demonstrated a maximum OD of 0.29 after 22 hours incubation and a growth rate of $0.009 \mathrm{~h}^{-1}$. Similarly the strain expressing ESA_pESA3p05450 grew to a maximum OD of 0.27 after 11 hours and reached a growth rate of $0.003 \mathrm{~h}^{-1}$. While each of the proP homologues tested conferred osmotolerance in $E$. coli MKH13, it was observed that the strains expressing ESA_00673, ESA_02131 and ESA_pESA3p05450 demonstrated the highest levels of osmotolerance in an osmolyte rich environment.

b) Growth in defined media plus proline at elevated osmolarity Of the six strains tested, E. coli MKH13 expressing ESA_02131, ESA_01706, ESA_03328 and ESA_pESA3 p05450 demonstrated osmotolerance (growth above 4\% $\mathrm{NaCl}$ ) in defined media supplemented with proline. Each of the four osmotolerant strains grew to an $\mathrm{OD} \geq$ 0.17 at a $\mathrm{NaCl}$ concentration of $9 \%$. E. coli MKH13 expressing ESA_02131 demonstrated the highest growth rate of the osmotolerant strains $\left(0.007 \mathrm{~h}^{-1}\right)$, reaching a final $\mathrm{OD}$ of 0.219 at $6 \% \mathrm{NaCl}$ (Table 3). While the growth rates of E. coli MKH13 expressing ESA_02131 in complex media are higher than those grown in defined media supplemented with proline there is also a significant difference in the maximum OD values recorded, with E. coli MKH13::ESA_02131 appearing to favour the osmolyte rich environment over media where proline is the only available compatible solute present.

c) Growth in defined media plus betaine at elevated osmolarity While E. coli MKH13 expressing ESA_00673 failed to grow above $4 \% \mathrm{NaCl}$, each of the five remaining strains demonstrated osmotolerance in defined media supplemented with betaine. E. coli MKH13::ESA_00673 also failed to grow above $4 \% \mathrm{NaCl}$ in defined media supplemented with $1 \mathrm{mM}$ proline but demonstrated an ability to grow in LB supplemented with $8 \%$ added $\mathrm{NaCl}$. E. coli MKH13::04214 demonstrated a growth rate of $0.013 \mathrm{~h}^{-1}$ at $5 \% \mathrm{NaCl}$ in betaine supplemented media but did not grow above $4 \% \mathrm{NaCl}$ in proline supplemented media. E. coli MKH13 expressing ESA_02131, ESA_01706, ESA_03328 and ESA_pESA3p05450 demonstrated the highest osmotolerance in defined media supplemented with betaine, growing up to $9 \%$ $\mathrm{NaCl}$. However, significantly higher growth rates and/or OD values were recorded for E. coli MKH13 expressing ESA_02131, ESA_01706 and ESA_03328 when compared to growth in proline supplemented media (Table 3 ).

\section{Discussion}

The adaptation of bacteria to increased osmotic stress involves the intracellular accumulation of organic compounds called osmolytes or compatible solutes (owing to their compatibility with vital cellular processes at high 
internal concentrations [19-21]). The main function of compatible solutes is to increase cell turgor, thus counterbalancing the external osmotic upshift and preventing water loss, which left unchecked leads to plasmolysis and ultimately cell death. The principal compatible solutes accumulated by $E$. coli under osmotic stress conditions are proline and betaine [22]. The osmoprotective properties of proline were first described by Christian in 1955 who reported that the addition of this amino acid to media at elevated osmolarity prevented bacterial growth inhibition [23]. Likewise, the trimethylamino acid glycine betaine has been extensively studied for its osmoprotective properties [24]. Betaine and proline uptake in $E$. coli is mediated primarily by the secondary uptake system ProP [25].

Previous in silico analysis revealed 53 putative osmotolerance loci on the C. sakazakii BAA-894 genome. Most notably, seven homologues of the E. coli proP gene were identified, each of which was believed to encode a transmembrane protein capable of transporting proline and/ or betaine [11]. Further analysis of each ProP homologue revealed structural similarities to the $E$. coli ProP protein with the gene encoding the most obvious ProP candidate ESA_02131 (based on overall\% identity), encoding the characteristic 12 trans-membrane domains, an extended central hydrophilic loop and a carboxy-terminal extension $[11,26]$. Interestingly, although the remaining six proP homologues exhibit significant sequence identity, they are all 60-70 amino acids shorter than ESA_02131 at the C-terminal end. Furthermore, the central hydrophilic loop of the E. coli proP is extended and this is a feature common to the protein encoded by the C. sakazakii ESA_02131 gene; however sequence analysis has revealed that although a central hydrophilic loop is present in each of the six additional proP homologues this periplasmic loop is not extended to the same degree as ESA_02131 (7-18 amino acids longer in ESA_02131 than other proP homologues identified).

In the current study we investigated the physiological role, particularly in response to salt tolerance, of the ProP porters identified previously in C. sakazaki. Firstly, in order to determine if the proP homologues are upregulated at the transcriptional level in response to an osmotic upshift, gene expression analysis was performed using the C. sakazakii BAA-894 strain. Interestingly, all six proP genes analyzed (ESA_02131, ESA_01706, ESA_04214, ESA_00673, ESA_03328 and ESAp_3p05450) demonstrated increased expression levels relative to the constitutively expressed 16 s gene (ESA_03798) when subject to an osmotic upshift; however expression levels varied significantly amongst the individual proP homologues (Figure 1). Surprisingly, the homologue which exhibited the highest level of upregulation following osmotic stress was not ESA_02131, the closest homologue to $E$. coli proP in terms of sequence identity, but rather ESA_04214. If we consider that ProP activity in E. coli has been shown to be regulated at the translational level via ProQ, this may go some way to explaining this incongruity [27]. ProQ is a cytoplasmic protein which was previously believed to play a role in post-translational modification of ProP; influencing the osmotic activation of this compatible solute uptake protein $[28,29]$. However, more recently, further analysis into the role of ProQ was warranted by the fact that proP transcript levels are unchanged in a proQ mutant, ProP expression is affected by deletion of pro $Q$ and no physical interaction was detected between ProP and ProQ [30]. As a result the role of ProQ was recently clarified as a translational regulator of proP mRNA. ProQ is a ribosome association protein, interacting with proP mRNA transcribed from both RpoD and RpoS promoters. As yet very little is known about the mechanism of ProQ mediated translation, however a deeper analysis of this protein has revealed a high affinity RNA binding domain and an RNA strand exchange and duplexing domain similar to the translational regulator FinO $[27,30]$. It is likely that, as with $E$. coli, ProP mediated osmotolerance is also regulated at the translational level in C. sakazakii, a factor which may account for our observed disconnect between transcriptional data and observed osmotolerance phenotype as outlined below. In support of this proposal, we have identified a ProQ homologue (ESA_01419) on the C. sakazakii BAA-894 genome.

Physiological analysis of E.coli MKH13 transformed with each C. sakazakii proP homologue reveals that six of the proP homologues initially identified in silico play a role in cellular osmoregulation in E. coli MKH13, a strain which is deficient in the compatible solute transporters putP, proP and proU rendering it sensitive to hyperosmotic conditions. Significant differences in growth rates were exhibited among strains grown in different environments where compatible solute availability varied. In particular, E. coli MKH13 expressing ESA_01706 failed to grow above $5 \%$ added $\mathrm{NaCl}$ when grown in LB (Figure 2), yet in minimal media with proline as the only available compatible solute this strain grew in the presence of up to $9 \% \mathrm{NaCl}$ (growth rate of $0.005 \mathrm{~h}^{-1}$ ). If we consider that competitive inhibition among osmolytes has been demonstrated previously and the osmoprotective effects of osmolytes varies significantly then these results are to be expected [31,32]. Indeed, inhibition of glycine betaine uptake by other compatible solutes, or indeed glycine betaine analogues, is a phenomenon which has been observed previously [32]. Such structural similarities and differing osmoprotective ranges may offer a plausable explanation for the increased growth rates observed in this study in defined media (where there is only one compatible solute - proline or glycine betaine) in comparison to complex media rich in 'competing' osmoprotective compounds. 

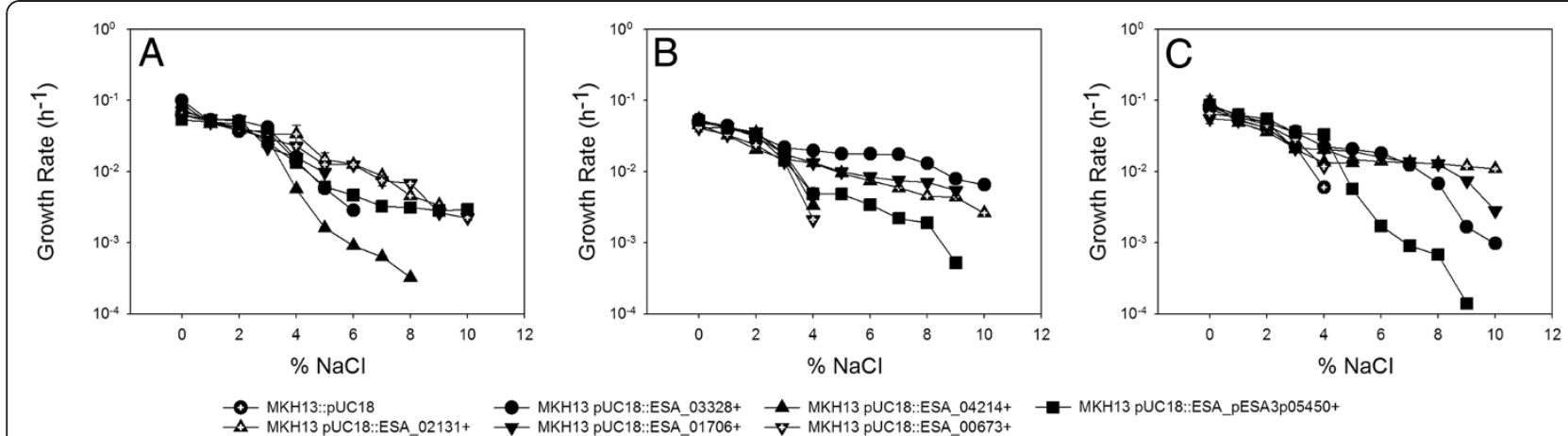

Figure 2 Determination of the osmotolerance of E. coli MKH13 strains transformed with proP homologues from C. sakazakii BAA-894 in A) complex media, B) defined media supplemented with proline and C) defined media supplemented with betaine.

While many studies have previously focused on the importance of the ProP porter to E. coli osmotolerance, we are the first to demonstrate importance of ProP activity in the osmotolerance of the neonatal pathogen C. sakzakii. Indeed, each of the ProP porters analysed conferred osmotolerance when expressed in E. coli MKH13, albeit to varying degrees depending on compatible solute availability. Interestingly, glycine betaine appeared to be the most osmoprotective compound outperforming proline. Five of the strains expressing a proP homologue grew above $4 \%$ $\mathrm{NaCl}$ in betaine supplemented media in comparison to four strains demonstrating growth above $4 \% \mathrm{NaCl}$ in proline supplemented media. Furthermore, growth rates of $E$. coli MKH13 expressing ESA_02131 and ESA_01706 were significantly higher in media supplemented with glycine betaine in comparison to proline (Table 3). Indeed it is likely that some of the transporters may be demonstrating specificity for one osmolyte over another but may transport both due to the structural similarities of proline and betaine. Another interesting finding was that ESA_00673 showed the highest osmotolerance of all the strains tested in $\mathrm{LB}$, yet did not grow above $4 \% \mathrm{NaCl}$ in defined media supplemented with either proline or glycine betaine suggesting that this porter might well be transporting an as yet unidentified osmolyte other than betaine or proline.

As outlined previously ESA_02131 was the most closely related homologue to the well characterized proP of E. coli and exhibited the characteristic $C$-terminal extension which is lacking in the other C. sakazakii proP homologues. The role of the carboxy-terminal extension in osmotolerance was defined by Culham et al. [33] when a derivative with alterations to specific amino acids on the C-terminal extension required a larger osmotic upshift for activation, when compared to the wild-type. The C-terminal domain has been implicated in osmosensing by other transporters such as BetP and OpuA in which osmolality controls the interaction of the C-terminal domain with each transporter, switching the transporter between active and inactive confirmations [34]. However, the C-terminal domain in
ProP does not appear to be required for activation of the protein as orthologues without the coiled-coil domain of the C-terminal extension still function as osmosensory transporters [35]. That being said, research has shown that a higher osmolality is required to activate transporters lacking the coiled-coil [35]. The lack of this highly sensitive coiled-coil domain in the C. sakazakii ProP homologues may be a consequence of $C$. sakazakii existing in more extreme osmotic environments when compared to $E$. coli which depends on a single ProP which is highly tuned by the coiled coil. Indeed Tsataski et al. [35] have previously demonstrated that the osmotic activation of the transporter ProP of E. coli is tuned by its C-terminal coiled-coil and osmotically induced changes in the phospholipid composition.

\section{Conclusion}

In the current study we have confirmed that six of the previously identified proP homologues play a role in the osmotolerance response of $C$. sakazakii. Gene expression analysis of each of these $\mathrm{proP}$ homologues demonstrated an increase in expression levels during an osmotic upshift. Furthermore, heterologous expression against an osmotically sensitive $E$. coli host revealed that each of the six homologues conferred an osmotolerance phenotype, albeit to varying degrees, in growth media with differences in osmolyte availability. The degeneracy of ProP porters in C. sakazakii goes some way to explaining the exteme osmotolerance phenotype of the bacterium, a factor which contributes significantly to its ability to survive in PIF. To date ProP in E.coli has been well characterized however this is the first study to demonstrate the importance of ProP in C. sakazakii osmotolerance. Knowledge of the transcriptional, translational and posttranslational regulation of these uptake systems will aid the future design and development of novel control stratagies for dealing with the pathogen, particularly in foods destined for neonatal consumption. Indeed, the use of small molecules to structurally mimic compatible solutes (smugglin technology) may be a viable alternative to antibiotics for the control of this pathogen in high risk foods such as powdered infant formula [36-38]. 


\section{Competing interests}

The authors declare that they have no competing interests.

\section{Authors' contributions}

AF and CJ carried out the molecular cloning. RG participated in the real-time analysis. AF also carried out the physiological analysis and real-time analysis and drafted the manuscript together with RDS. All authors read and approved the final manuscript.

\section{Acknowledgements}

R.D.S. is Coordinator of the EU FP7 ClouDx-i project. A.F. is funded by an IRCSET EMBARK Postgraduate Scholarship RS/2010/2300.

Received: 9 April 2014 Accepted: 14 May 2014

Published: 24 May 2014

\section{References}

1. Iversen C, Lehner A, Mullane N, Bidlas E, Cleenwerck I, Marugg J, Fanning S, Stephan $\mathrm{R}$, Joosten $\mathrm{H}$ : The taxonomy of Enterobacter sakazakii: proposal of a new genus Cronobacter gen. nov. and descriptions of Cronobacter sakazakii comb. nov. Cronobacter sakazakii subsp. sakazakii, comb. nov., Cronobacter sakazakii subsp. malonaticus subsp. nov., Cronobacter turicensis sp. nov., Cronobacter muytjensii sp. nov., Cronobacter dublinensis sp. nov. and Cronobacter genomospecies 1. BMC Evol Biol 2007, 7(1):64.

2. Joseph S, Forsythe S: Insights into the emergent bacterial pathogen Cronobacter spp., generated by multilocus sequence typing and analysis. Front Microbiol 2012, 3:1-11.

3. Brady C, Cleenwerck I, Venter S, Coutinho T, De Vos P: Taxonomic evaluation of the genus Enterobacter based on multilocus sequence analysis (MLSA): Proposal to reclassify E. nimipressuralis and E. amnigenus into Lelliottia gen. nov. as Lelliottia nimipressuralis comb. nov. and Lelliottia amnigena comb. nov., respectively, E. gergoviae and E. pyrinus into Pluralibacter gen. nov. as Pluralibacter gergoviae comb. nov. and Pluralibacter pyrinus comb. nov., respectively, E. cowanii, E. radicincitans, E. oryzae and E. arachidis into Kosakonia gen. nov. as Kosakonia cowanii comb. nov., Kosakonia radicincitans comb. nov., Kosakonia oryzae comb. nov. and Kosakonia arachidis comb. nov., respectively, and E. turicensis, E. helveticus and E. pulveris into Cronobacter as Cronobacter zurichensis nom. nov., Cronobacter helveticus comb. nov. and Cronobacter pulveris comb. nov., respectively, and emended description of the genera Enterobacter and Cronobacter. Syst Appl Microbiol 2013, 36(5):309-319.

4. Friedemann M: Epidemiology of invasive neonatal Cronobacter (Enterobacter sakazakii) infections. Eur J Clin Microbiol Infect Dis 2009, 28(11):1297-1304.

5. Nazarowec-White M, Farber JM: Enterobacter sakazakii: a review. Int J Food Microbiol 1997, 34(2):103-113.

6. Forsythe SJ: Enterobacter sakazakii and other bacteria in powdered infant milk formula. Matern Child Nutr 2005, 1(1):44-50.

7. Friedemann M: Enterobacter sakazakii in food and beverages (other than infant formula and milk powder). Int J Food Microbiol 2007, 116(1):1-10.

8. Nazarowec-White M, Farber JM: Incidence, survival, and growth of Enterobacter sakazakii in infant formula. J Food Prot 1997, 60(3):226-230.

9. Caubilla Barron J, Forsythe SJ: Dry stress and survival time of Enterobacter sakazakii and other Enterobacteriaceae in dehydrated powdered infant formula. J Food Prot 2007, 70(9):2111-2117.

10. Al-Nabulsi AA, Osaili TM, Al-Holy MA, Shaker RR, Ayyash MM, Olaimat AN, Holley RA: Influence of desiccation on the sensitivity of Cronobacter spp. to lactoferrin or nisin in broth and powdered infant formula. Int J Food Microbiol 2009, 136(2):221-226.

11. Feeney A, Sleator RD: An in silico analysis of osmotolerance in the emerging gastrointestinal pathogen Cronobacter sakazakii. Bioeng Bugs 2011, 2(5):260-270.

12. Kucerova E, Clifton SW, Xia XQ, Long F, Porwollik S, Fulton L, Fronick C, Minx P, Kyung K, Warren W, Fulton R, Feng D, Wollam A, Shah N, Bhonagiri V, Nash WE, Hallsworth-Pepin K, Wilson RK, McClelland M, Forsythe SJ: Genome sequence of Cronobacter sakazakii BAA-894 and comparative genomic hybridization analysis with other Cronobacter Species. PLoS One 2010, 5(3):e9556.
13. Vieira J, Messing J: The pUC plasmids, an M13mp7-derived system for insertion mutagenesis and sequencing with synthetic universal primers. Gene 1982, 19(3):259-268.

14. Baldwin A, Loughlin M, Caubilla-Barron J, Kucerova E, Manning G, Dowson C, Forsythe S: Multilocus sequence typing of Cronobacter sakazakii and Cronobacter malonaticus reveals stable clonal structures with clinical significance which do not correlate with biotypes. BMC Microbiol 2009, 9(1):223.

15. Kempf B, Bremer E: OpuA, an osmotically regulated binding proteindependent transport system for the Osmoprotectant Glycine Betaine in Bacillus subtilis. J Biol Chem 1995, 270(28):16701-16713.

16. Walsh $\mathrm{P}$, Carroll J, Sleator RD: Accelerating in silico research with workflows: a lesson in simplicity. Comput Biol Med 2013, 43(12):2028-2035.

17. Sambrook J, Fritsch EF, Maniatis T: Molecular Cloning A Laboratory Manual, vol. 1-3. New York: Cold Spring Harbor Laboratory Press; 1989.

18. Livak KJ, Schmittgen TD: Analysis of relative gene expression data using Real-Time Quantitative PCR and the 2- $\Delta \Delta C T$ method. Methods 2001, 25(4):402-408.

19. Sleator RD, Hill C: Bacterial osmoadaptation: the role of osmolytes in bacterial stress and virulence. FEMS Microbiol Rev 2002, 26(1):49-71.

20. Sleator RD, Gahan CG, Hill C: A postgenomic appraisal of osmotolerance in Listeria monocytogenes. App/ Environ Microbio/ 2003, 69(1):1-9.

21. Hill C, Cotter PD, Sleator RD, Gahan CGM: Bacterial stress response in Listeria monocytogenes: jumping the hurdles imposed by minimal processing. Int Dairy J 2001, 12:273-283.

22. Milner JL, McClellan DJ, Wood JM: Factors reducing and promoting the effectiveness of proline as an Osmoprotectant in Escherichia coli K12. J Gen Microbiol 1987, 133(7):1851-1860.

23. Christian J: The influence of nutrition on the water relations of Salmonella Oranienburg. Aust J Biol SCi 1955, 8(1):75-82.

24. Sleator RD, Gahan CGM, Abee T, Hill C: Identification and disruption of BetL, a secondary Glycine Betaine transport system linked to the salt tolerance of Listeria monocytogenes LO28. Appl Environ Microbiol 1999, 65(5):2078-2083.

25. Culham DE, Lasby B, Marangoni AG, Milner JL, Steer BA, van Nues RW, Wood JM: Isolation and sequencing of Escherichia coli Gene proP reveals unusual structural features of the Osmoregulatory Proline/Betaine transporter, ProP. J Mol Biol 1993, 229(1):268-276.

26. Sleator RD: Proteins: form and function. Bioeng Bugs 2012, 3(2):80-85.

27. G Chaulk S, Smith-Frieday MN, Arthur DC, Culham DE, Edwards RA, Soo P, Frost LS, Keates RAB, Glover JNM, Wood JM: ProQ Is an RNA Chaperone that controls ProP levels in Escherichia coli. Biochemistry 2011, 50(15):3095-3106.

28. Milner JL, Wood JM: Insertion proQ220::Tn5 alters regulation of proline porter II, a transporter of proline and glycine betaine in Escherichia coli. J Bacteriol 1989, 171(2):947-951.

29. Kunte HJ, Crane RA, Culham DE, Richmond D, Wood JM: Protein ProQ influences osmotic activation of compatible solute transporter ProP in Escherichia colik-12. J Bacteriol 1999, 181(5):1537-1543.

30. Sheidy DT, Zielke RA: Analysis and expansion of the role of the Escherichia coli Protein ProQ. PLoS One 2013, 8(10):e79656.

31. Sleator RD, Gahan CGM, Hill C: Identification and disruption of theproBA Locus in Listeria monocytogenes: Role of Proline Biosynthesis in salt tolerance and murine infection. Appl Environ Microbiol 2001, 67(6):2571-2577.

32. Mendum ML, Smith LT: Characterization of Glycine Betaine Porter I from Listeria monocytogenes and its roles in salt and chill tolerance. Appl Environ Microbiol 2002, 68(2):813-819.

33. Culham DE, Tripet B, Racher KI, Voegele RT, Hodges RS, Wood JM: The role of the carboxyl terminal a-helical coiled-coil domain in osmosensing by transporter ProP of Escherichia coli. J Mol Recognit 2000, 13(5):309-322.

34. Keates RAB, Culham DE, Vernikovska YI, Zuiani AJ, Boggs JM, Wood JM: Transmembrane Helix I and Periplasmic Loop 1 of Escherichia coli ProP Are Involved in Osmosensing and Osmoprotectant Transport. Biochemistry 2010, 49(41):8847-8856.

35. Tsatskis Y, Khambati J, Dobson M, Bogdanov M, Dowhan W, Wood JM: The osmotic activation of transporter ProP Is tuned by both its C-terminal Coiled-coil and Osmotically induced changes in phospholipid composition. J Biol Chem 2005, 280(50):41387-41394. 
36. Sleator RD, Hill C: Food reformulations for improved health: a potential risk for microbial food safety? Med Hypotheses 2007, 69(6):1323-1324.

37. Sleator RD, Hill C: New frontiers in probiotic research. Lett Appl Microbiol 2008, 46(2):143-147.

38. Sleator RD, Banville N, Hill C: Carnitine enhances the growth of Listeria monocytogenes in infant formula at 7 degrees C. J Food Prot 2009, 72(6):1293-1295

doi:10.1186/1757-4749-6-15

Cite this article as: Feeney et al: Analysis of the role of the Cronobacter sakazakii ProP homologues in osmotolerance. Gut Pathogens 2014 6:15.

\section{Submit your next manuscript to BioMed Central and take full advantage of:}

- Convenient online submission

- Thorough peer review

- No space constraints or color figure charges

- Immediate publication on acceptance

- Inclusion in PubMed, CAS, Scopus and Google Scholar

- Research which is freely available for redistribution 